"Hospital Naval "Almirante Nef". Viña del Mar, Chile.

${ }^{2}$ Academia de Humanidades, Universidad San Sebastián. Santiago, Chile.

${ }^{3}$ Facultad de Medicina, Universidad de Chile. Santiago, Chile.

${ }^{4}$ Clínica Santa María. Santiago, Chile.

${ }^{5}$ Centro de Bioética, Facultad de Medicina Pontificia Universidad Católica de Chile. Santiago, Chile.

${ }^{6}$ Facultad de Medicina, Universidad de los Andes. Santiago, Chile

${ }^{7}$ Clínica Las Condes. Santiago, Chile.

${ }^{8}$ Facultad de Medicina, Universidad Andrés Bello. Viña del Mar, Chile.

Recibido el 9 de julio de 2021, aceptado el 10 de agosto de

Correspondencia a:

Dr. Carlos Echeverría B. Hospital Naval "Almirante Nef", Subida Alessandri s/n. Viña del Mar, Chile. ciecheverria@hotmail.com

\section{Una reflexión ética sobre la telemedicina}

\author{
GRUPO DE ESTUDIOS DE ÉTICA CLÍNICA DE LA \\ SOCIEDAD MÉDICA DE SANTIAGO \\ MIEMBROS: CARLOS ECHEVERRÍA B. ${ }^{1}$ (Presidente), \\ ALBERTO ROJAS O. ${ }^{1}$, ALEJANDRO SERANI M. ${ }^{2}$, \\ ANAMARÍA ARRIAGADA U. ${ }^{3}$, GONZALO RUIZ-ESQUIDE ${ }^{4}$, \\ RODRIGO SALINAS R. ${ }^{3}$, PAULINA TABOADA R. ${ }^{5}$, \\ CARLOS QUINTANA V. ${ }^{6}$, RICARDO VACAREZZA Y. ${ }^{3}$, \\ MARÍA A. RODRÍGUEZ S. ${ }^{7}$, HERNÁN BORJA R. ${ }^{8}$
}

\section{A reflection about the ethical implications of telemedicine}

Recognizing the role of technology in the development of medicine and the impact of telecommunication advances, we reflect on the meaning and ethics of the use of Telemedicine, both in its general dimension for the use and distribution of knowledge, as well as in the delivery of health actions, scientific research, and data management. Teleconsultation is discussed in greater detail, analyzing its process and application, reviewing its possible advantages and disadvantages, from the point of view of providers and patients. We highlight the need to carry out an appropriate evaluation of each instance, from the point of view of both the patient and the professional who uses it. The importance of maintaining a doctor-patient relationship in agreement with the nature and practice of Medicine, respecting people's dignity, is emphasized. We mention the ethical conditions that must be bore in mind for the proper use of telemedicine. We discuss the eventual influence that this practice will have on the concept and practice of medical care, while suggesting the need to legislate on the matter.

(Rev Med Chile 2021; 149: 928-933)

Key words: Ethical Analysis; Physician-Patient Relations; Remote Consultation; Telemedicine.

\section{La Medicina y el desarrollo tecnológico}

\section{Grupo de Estudios de Ética Clínica de la Sociedad Médica de Santiago}

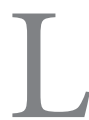

os avances de la ciencia en la historia de la Medicina son innumerables, favoreciendo a la salud individual, y también la colectiva. Los conocimientos y tecnologías derivadas de estos avances han permitido optimización en la prevención, diagnósticos y cada vez mejores tratamientos. No obstante, ese desarrollo no siempre ha significado resultados deseables. Incluso puede ser discutible la eticidad de su puesta en práctica, como por ejemplo en el caso de la clonación humana.

Este trabajo pretende reflexionar sobre la bondad y uso apropiado de la telemedicina en la práctica médica, no solo por el hecho que ella sea factible tecnológicamente, sino considerando que, en toda intervención en la salud de una persona, no sólo está en juego la posibilidad técnica, sino que además ella debe cumplir con criterios éticos aceptables. 


\section{¿Qué es telemedicina?}

El tema es relativamente reciente y vincula directamente la aplicación e impacto de las tecnologías de la comunicación en prestaciones de salud. Field ${ }^{1}$ señala que la telemedicina (TM) consiste en el uso de información electrónica y tecnologías de la comunicación para proveer y dar soporte al cuidado de la salud, siendo la distancia entre los usuarios un factor a considerar. Él estimaba que el campo de aplicación era muy amplio, extendiéndose desde el uso del teléfono para consultas hasta asesoría para efectuar cirugía remota experimental.

La Organización Mundial de la Salud adoptó la siguiente definición para esta materia:

"La prestación de servicios de atención de la salud, donde la distancia es un factor crítico, por todos los profesionales de la salud que utilizan tecnologías de la información y de la comunicación para el intercambio de información válida para el diagnóstico, tratamiento y prevención de enfermedades y lesiones, la investigación y la evaluación, y para la formación continuada de los profesionales de la salud, todo en aras de avanzar en la salud de los individuos y sus comunidades".

En nuestro medio, Lozano ${ }^{3}$ sostiene que "la telemedicina es el resguardo u optimización de la continuidad del cuidado mediante herramientas de tecnologías de la información y comunicaciones que hacen posible al menos una acción remota durante el proceso. Se refiere al conjunto completo de prestaciones y servicios que pueden brindarse en forma remota y tienen como objetivo mejorar el nivel de salud de una población mediante la optimización de la cadena del cuidado".

Ante tantas posibles aplicaciones, algunas de potencial impacto positivo en la salud personal o colectiva, es preciso analizar si se trata solamente de la optimización de una tecnología particular o hay razones para sostener que es un desarrollo útil y necesario para las acciones en salud.

Dado que el avance en las comunicaciones será cada vez más rápido, así como su influencia en la vida diaria (v.gr. el paso de redes telefónicas analógicas a las digitales, el uso de $3 \mathrm{G}$ a $4 \mathrm{G}$ y $5 \mathrm{G}^{4}$ ), resulta necesaria la reflexión de su vinculación con la medicina.

\section{Algunos factores a considerar:}

a) Existen condiciones que favorecen el desarrollo de la Telemedicina, como el notable desarrollo de tecnologías de transmisión y comunicación de datos, cada vez más accesible a la ciudadanía, las derivadas de la digitalización de imágenes o la creación de bases de datos y técnicas que permiten mayor acceso a un conocimiento rápidamente cambiante y de gran volumen;

b) Las dificultades de acceso a determinadas prestaciones de salud por ruralidad, razones socio económicas, escasez de especialistas a nivel local, entre otros;

c) El deseo social de obtener más y mejores atenciones en salud;

d) El interés de prestadores de la salud para que sus acciones sean de la mayor cobertura posible y, desde el punto de vista de los pagadores o aseguradores, lograr menores costos.

\section{Posibles niveles de aplicación:}

La Telemedicina puede ser útil en diversos ámbitos:

\section{a) Adquisición, almacenamiento, concentración y difusión del conocimiento}

Hoy día es factible acceder a bibliotecas digitales que, respetando la normativa legal, pueden ser puestas a disposición de profesionales de la salud para consulta y aprendizaje.

También es posible crear bases de datos interactivas, que permitan difundir distintas materias, como también revisiones actualizadas sobre ellas. Tal es el caso de UpToDate, destinado a dar soporte a las decisiones clínicas utilizando evidencias, o Epistemonikos ${ }^{5}$, una base de datos colaborativa multilingüe de evidencia en salud, o aplicaciones móviles como The Radiology Assistant ${ }^{6}$ o Epocrates $^{7}$, entre otras.

Un desarrollo particular lo constituyen las conferencias virtuales sobre temas específicos, participar en consejería de pequeños grupos mejorando sus conocimientos y habilidades, así como reuniones clínicas y similares, incluyendo la discusión de casos clínicos. Ello permite asesorar en la toma de decisiones acerca de los mismos.

\section{b) Otorgamiento de prestaciones de salud}

La técnica permite acceso telemático a consultas médicas, e informes de exámenes como fondo de ojo, estudios de imágenes, laboratorio y otros. 
Por su trascendencia, el punto se desarrolla más adelante.

\section{c) Investigación científica aplicada a las enfermedades y tecnologías sanitarias}

Se refiere a la búsqueda y adquisición de información, acorde con el método científico, destinadas a obtener o perfeccionar el conocimiento y/o evaluar los resultados obtenidos con distintas acciones de salud, a nivel individual o de poblaciones, además de analizar la eficiencia y eficacia de diversas prestaciones, o la utilización de tecnologías que pretenden resolver problemas sanitarios.

\section{d) Obtención y manejo de datos}

Estos son personales y/o clínicos de los pacientes. Su disposición y almacenamiento es esencial para el manejo administrativo y la atención de los pacientes.

Existen diversos software informáticos, que permiten individualizar a los usuarios, así como el registro de los servicios solicitados. Se facilita saber a qué prestaciones tienen derecho de acuerdo a sus contratos o disposiciones legales, gestionar los recursos que se utilizan en las acciones de salud, incluyendo pagos, aportes personales, licencias médicas y otros documentos que la legislación y normas respectivas demanden.

Se incluye desde el valor de un examen de laboratorio, resultados de procedimientos diagnósticos, terapéuticos, imágenes, historia clínica, generación de Grupos Relacionados por Diagnóstico $(\mathrm{GRD})^{8}$ para análisis y comparación económica, hasta el beneficio que puede traer aparejado la utilización de inteligencia artificial.

La llamada "inteligencia artificial" procura la optimización de los diagnósticos -y eventualmente-tratamientos. Su aplicación, mediante los denominados sistemas expertos (que pretenden emular el razonamiento humano y generar un desempeño como el de "expertos en la materia") u otros, con variados flujogramas, intentan mejorar las posibilidades de éxito. Esto puede ser relevante para resolver ciertos problemas diagnósticos, con algoritmos unidos a bases de datos significativas y evidencia científica para mejorar el proceso diagnóstico o terapéutico. En otro plano, tiene un rol en intervenciones quirúrgicas con ayuda de la robótica'.

Sin embargo, existen limitaciones derivadas de la concepción, ejecución y eventuales sesgos en los algoritmos ${ }^{10}$, como el peligro de dejar de lado la atención integral de las personas.

Debido a la amplitud de los temas en este ámbito, nos abocaremos fundamentalmente a la teleconsulta.

\section{La teleconsulta y el acto médico}

\section{Limitaciones y riesgos}

Mesa y Pérez ${ }^{11}$ han planteado que es posible el acto médico, en el contexto de la telemedicina, cuando se cumplen ciertas condiciones. Por ello nos parece que esa afirmación deba ser cuidadosamente considerada al utilizar esta herramienta, para respetar la naturaleza y sentido de las prestaciones médicas, así como la dignidad de los pacientes. Examinaremos los siguientes tópicos:

- La teleconsulta tiene las limitaciones inherentes a la distancia e interposición de un elemento ajeno y distractor como es el sistema comunicacional y computacional que la hace posible.

- Desde el punto de vista de la práctica médica, modifica la interacción entre el médico y el paciente, al no haber examen físico y el contacto cercano, necesarios en la evaluación clínica, aumentando la posibilidad de error. Por ello parece adecuado establecer claramente el objeto perseguido y sus limitaciones.

- El paciente debe ser advertido, a través de un consentimiento informado, de las limitaciones del procedimiento. Aun así, éste puede no tener la suficiente libertad personal para aceptar o no el sistema propuesto. Al no tener acceso concreto a un especialista, pero disponer de él por vía remota, el paciente puede optar por lo que estime como "el mal menor". Sin embargo, debe considerarse que esta decisión, determinada por las circunstancias, puede no ser genuinamente libre, sino estar severamente condicionada por la situación. Surge el peligro que se estimule esta modalidad de atención, prestando atención únicamente a la facilidad de acceso o criterios económicos.

- Aunque se trate de condiciones habituales o de excepción, una atención de telemedicina no debe admitirse de una calidad inferior a la que razonablemente se busca. 
- Existe el riesgo de la pérdida o vulneración de la confidencialidad, sea por la presencia necesaria de otros actores, como mediadores para la teleconsulta, o por filtraciones durante el tránsito de los datos, en cualquier sentido. Particular cuidado representa el sitio donde se efectúa el procedimiento y las limitaciones físicas (por ejemplo, de vista u oído) que pueda tener el consultante.

- Por otra parte, al generalizar el procedimiento de teleconsulta, ésta requiere la disponibilidad de equipos y líneas de comunicación eficientes, que en distintas zonas del país no están disponibles, ya sea por problemas de infraestructura o por limitaciones económicas y culturales de los pacientes. Además, los equipos de comunicación llamados "teléfonos inteligentes", son caros y difíciles de operar, especialmente para los pacientes mayores y en zonas alejadas, donde la disposición de estos aparatos no ha penetrado suficientemente.

- Cada consulta debería ser evaluada objetivamente para determinar si ha cumplido con el objetivo propuesto, e informar al consultante la necesidad de controles o consultas presenciales.

\section{Otros elementos a mencionar}

En otras situaciones, como por ejemplo al fotografiar una lesión, para ser interpretada por un especialista a distancia, ello será verdaderamente útil si se cuenta con recursos tecnológicos de calidad que impidan las distorsiones y, por ende, el error. El inconveniente es que el trabajo del "lector" puede transformarse en un proceso automatizado de análisis de fotografías, desdibujando lo propiamente humano que caracteriza a la atención médica. Adicionalmente, el paciente puede verse privado de la posibilidad de hacer preguntas, o ellas pudieran ser contestadas por un tercero no especialista. Este hecho impide una buena relación médico-paciente. Es necesario que los propios especialistas analicen y definan apropiadamente los requisitos técnicos que deben cumplirse para la correcta atención de los pacientes, buscando calidad de la prestación, el mejor diagnóstico posible, y la dignidad de las personas. Se espera que trabajos prospectivos puedan contribuir a la validación del método.

La ausencia de examen físico es una limitación severa en el contexto de una consulta médica, aun cuando existan -eventualmente-dispositivos que permitan auscultar a un paciente por vía remota, por ejemplo. La ausencia de cercanía física dificulta al médico valorar la actitud corporal, el estado psicológico del paciente y entablar una relación de cordialidad y confianza, elementos que son esenciales para una relación médico paciente satisfactoria. Si bien en un control posterior la teleconsulta puede ser un método utilizable, no debiera, en lo posible, ser un procedimiento de elección para una primera oportunidad de contacto con el paciente.

Debido a los requerimientos derivados de su modalidad tecnológica, la teleconsulta puede ser evaluada como de menor costo, tanto humano como material, ya que permite contactar, por ejemplo, a un menor número de especialistas con un mayor número de pacientes. Esta situación puede significar que se confíe en medidas basadas en eficiencia económica, desatendiendo el desarrollo de ciertas especialidades o minimizando el contacto de los pacientes con los médicos. De ser así, más que constituir un adelanto éticamente sustentable, puede terminar dañando seriamente el espíritu ancestral que caracteriza a la medicina, basada en la presencia concreta del médico y el equipo de salud, reemplazándolo por una relación de carácter mecanicista. Por otra parte, la atención médica orientada únicamente a la cobertura, puede originar el riesgo de ofrecer un servicio de teleconsulta aparentemente adecuado en lo formal, pero que en realidad solo está cumpliendo de forma parcial y carente de la calidad necesaria, generando una falsa sensación de atención. Este riesgo disminuye con una actitud prudente y consciente de las limitaciones por parte del médico y conocimiento de ellas por parte del enfermo.

Nos parece relevante que se obtenga el mayor beneficio posible, corrigiendo deficiencias que pudiesen llevar a considerar que la telemedicina sea reconocida como inadecuada y se desprestigie, como una despersonalización de la medicina, no contribuyendo a las necesarias soluciones y eficaz cobertura de problemas de salud.

\section{Evaluación general desde la ética}

La relación médico-paciente adecuada respeta a la persona y su dignidad y estimula su participación en la toma de decisiones.

Debe velarse por la congruencia y fiabilidad 
de los medios puestos a disposición de pacientes y médicos, así como la evaluación de objetivos y programas de la teleconsulta.

Respecto a datos administrativos y datos sensibles de los pacientes se deben tener en cuenta todas las precauciones necesarias para evitar su difusión no autorizada, bajo ninguna circunstancia, acorde a las normas y regulaciones sobre la materia ${ }^{12,13}$.

Debe existir particular cuidado en las etapas de transmisión de datos a distancia, buscando congruencia y fiabilidad de los mismos, como también en los registros, con procedimientos de seguimiento y control eficaces y seguros en el manejo de los datos.

Un tema importante es que los sistemas de comunicación involucrados en el proceso, sean eficaces, con infraestructura adecuada, que funcione en condición de emergencia u otras circunstancias. Los respaldos deben evitar la pérdida de datos.

Es preciso que el procedimiento sea debidamente comprendido por el paciente, y se otorgue en las mejores condiciones posibles, ya que se trata de un hecho privado. De ser necesario, se indicará una consulta presencial.

Es relevante comprender que la telemedicina no es una solución económico-administrativa para aumentar coberturas y soslayar carencias de especialistas, sino aprovechar sus beneficios, respetando sus limitaciones y resguardos éticos, clínicos y administrativos, resguardando en todo momento la dignidad de las personas.

\section{Las responsabilidades}

En el plano académico, debe enseñarse en forma real y adecuada esta modalidad de atención

En las opiniones emitidas en las reuniones clínicas, éstas pertenecen al opinante, pero la responsabilidad de la ejecución de un acto determinado, recae en el tratante.

Particularmente compleja es la asesoría de una situación clínica a distancia, sin ver ni examinar al paciente. Solo se dispone de comentarios y/o información obtenida a través terceros, que no poseen el entrenamiento para hacer una buena observación, por no ser personal sanitario, como es el caso de las consultas remotas en el ámbito marítimo. En esos casos, se debe informar claramente de las limitaciones de la consejería, siendo decisiva la obtención de los mejores antecedentes posibles, evitando riesgos hacia el paciente.

\section{Aspectos legales}

En este aspecto de la teleconsulta, se echa de menos una legislación que permita enfrentar, no solo el tema de las eventuales responsabilidades personales de quien actúa en el contexto, a veces confuso, de estas consultas, muchas veces en medio de mayor incertidumbre que una atención presencial. También se requiere un reglamento que especifique los requisitos para este tipo de atención médica.

\section{Conclusiones}

La teleconsulta constituye una situación de gran interés, trascendencia e impacto para la atención médica.

Es un avance tecnológico que puede significar un cambio sustantivo en la modalidad de atención médica, pero que debe ser utilizado adecuadamente, con conocimiento de sus limitaciones, manteniendo siempre una apropiada relación médico-paciente. Para ello debe asegurar su eficacia técnica, que manteniendo la naturaleza de la acción médica y la tradición hipocrática, logre los objetivos planteados, respetando la dignidad de las personas.

Una adecuada relación médico-paciente debe llevarse a cabo en forma presencial. La telemedicina representa una oportunidad para mejorar el acercamiento de la atención a grupos que no disponen de otra alternativa, comportándose como una herramienta complementaria, sin reemplazar cabalmente a lo presencial.

Debe evaluarse cada acción en términos de cumplimiento de objetivos, llevar a cabo estudios de control de calidad y seguimiento para conocer y medir la calidad de las prestaciones otorgadas, corregir deficiencias observadas y comunicar resultados

Adicionalmente, se estima necesario legislar sobre esta modalidad de atención.

El procedimiento debe ser adecuadamente explicado y comprendido por el paciente. Las condiciones técnicas y humanas deben resguardar la dignidad del mismo, reconociendo las limitaciones del método y mantener la confidencialidad del acto médico.

Particular atención requiere la calidad de la infraestructura y la tecnología que se utilizará, su seguridad y eficacia y las posibles limitaciones por factores socio-culturales. 
Debe disponerse de personal operador con condiciones morales y técnicas apropiadas, y resguardar el acceso, almacenamiento, disponibilidad, seguridad, integridad y confiabilidad de los datos comprometidos.

\section{Referencias}

1. Field MJ. Telemedicine: a guide to assessing telecommunications in healthcare. J Digit Imaging 1997; 10 (3 Suppl 1): 28. https://doi.org/10.1007/BF03168648.

2. OPS. OMS. PAHO/WHO eHealth Program. Marco de Implementación de un Servicio de Telemedicina. Washington DC, 2016. Disponible en: https://iris.paho.org/ bitstream/handle/10665.2/28413/9789275319031_spa. pdf?sequence $=6 \&$ isAllowed $=y \quad$ (Consultado el 26 de diciembre de 2010).

3. Lozano A. Guía práctica para la implementación de telemedicina. Septiembre 2020.

4. Primera licitación $5 \mathrm{G}$ en Latinoamérica. Disponible en: https://www.gob.cl/5g/ (Consultado el 5 de marzo de 2021).

5. Epistemonikos. Disponible en: https://www.epistemonikos.org/es

6. Wood LE, Picard MM, Kovacs MD. App Review: The
Radiology Assistant 2.0. J Digit Imaging 2018; 31: 383-6 https://doi.org/10.1007/s10278-018-0070-2.

7. Bhanot S, Sharma A. App Review Series: Epocrates. J Digit Imaging 2017; 30: 534-6. DOI 10.1007/s10278017-9977-2.

8. Águila A, Muñoz MA, Sepúlveda V. Experiencia en el desarrollo e implementación de la metodología de grupos relacionados por diagnóstico en un hospital universitario chileno. Evaluación a diez años de funcionamiento. Rev Med Chile 2019; 147: 1518-26.

9. Gandanglia G, Montorsi F, Karakiewicz PI, Sun M. Robot-assisted radical prostatectomy in prostate cáncer. Future Oncol. 2015; 11 (20): 2767-73.

10. Keskinbora KH. Medical ethics considerations on artificial intelligence. J Clin Neurosci. 2019; 64: 277-82.

11. Mesa M, Pérez I. El acto médico en la era de la telemedicina. Rev Med Chile 2020; 148: 852-7.

12. Ley 19628. Sobre protección de la vida privada. Ministerio Secretaría General de la Presidencia.Chile. Disponible en: https://www.bcn.cl/leychile/navegar?idNorma $=141599$ (Consultado el 20 de mayo de 2021).

13. Ley 20584. Regula los derechos y deberes que tienen las personas en relación con acciones vinculadas a su atención en salud 19628Ministerio de Salud; Chile. Disponible en: https://www.bcn.cl/leychile/navegar?idNorma=1039348 (Consultado el 20 de mayo de 2021). 